\title{
On the Structure of Graphs with Low Obstacle Number*
}

\author{
János Pach ${ }^{1,2}$, Deniz Sarıöz ${ }^{2}$ \\ 1 École Polytechnique Fédérale de Lausanne \\ Station 8, CH-1015 Lausanne, Switzerland \\ e-mail: pach@cims.nyu.edu \\ 2 The Graduate Center of the City University of New York \\ 365 Fifth Avenue, New York, NY 10016 USA \\ e-mail: sarioz@acm.org
}

\begin{abstract}
The obstacle number of a graph $G$ is the smallest number of polygonal obstacles in the plane with the property that the vertices of $G$ can be represented by distinct points such that two of them see each other if and only if the corresponding vertices are joined by an edge. We list three small graphs that require more than one obstacle. Using extremal graph theoretic tools developed by Prömel, Steger, Bollobás, Thomason, and others, we deduce that for any fixed integer $h$, the total number of graphs on $n$ vertices with obstacle number at most $h$ is at most $2^{o\left(n^{2}\right)}$. This implies that there are bipartite graphs with arbitrarily large obstacle number, which answers a question of Alpert, Koch, and Laison [1].
\end{abstract}

Key words. Obstacle number, visibility graph, hereditary graph property, forbidden induced subgraphs, split graphs, enumeration

Mathematics Subject Classification (1991): 05C62, 05C75, 68R10

\section{Introduction}

Consider a set $P$ of points in the plane and a set of closed polygonal obstacles whose vertices together with the points in $P$ are in general position, that is, no three of them are on a line. The corresponding visibility graph has $P$ as its vertex set, two points $p, q \in P$ being connected by an edge if and only if the segment $p q$ does not meet any of the obstacles. Visibility graphs are extensively studied and used in computational geometry, robot motion planning, computer vision, etc.; see [2], [8], [9], [10], [16].

Recently, Alpert, Koch, and Laison [1] introduced an interesting new parameter of graphs, closely related to visibility graphs. Given a graph $G$, we say that a set of points and a set of polygonal obstacles as above constitute an obstacle representation of $G$, if the corresponding visibility graph is isomorphic to $G$. A representation with $h$ obstacles is also called an $h$-obstacle representation. The smallest number of obstacles in an obstacle representation of $G$ is called the obstacle number of $G$.

Alpert et al. [1] showed that any representation of the bipartite graph $G_{1}$ which can be obtained by removing a maximum matching from a complete bipartite graph $K_{5,7}$, requires at least $t w o$ obstacles. They also constructed a split graph $G_{2}$, i.e., a graph that splits into a complete subgraph and an independent set, with a number of edges running between them, which has obstacle number at least two.

In Section 3, we complement the above examples with a third one: we construct a graph $G_{3}$ with obstacle number at least $t w o$, whose complement is a bipartite graph.

Lemma 1.1. There is a graph $G_{3}$ with obstacle number at least two, which consists of two complete subgraphs with a number of edges running between them.

\footnotetext{
* Research supported by NSA grant 47149-0001, NSF grant CCF-08-30272, Swiss National Science Foundation grant 200021-125287/1, and by the Bernoulli Center at EPFL.
} 


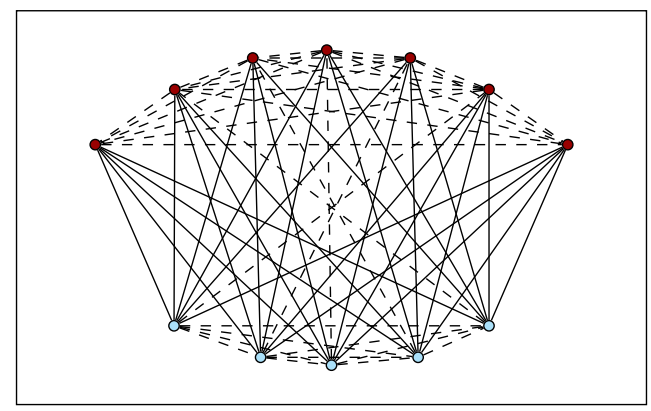

Fig. 1.1. A drawing of $G_{1}$ that can be completed to a 2-obstacle representation.

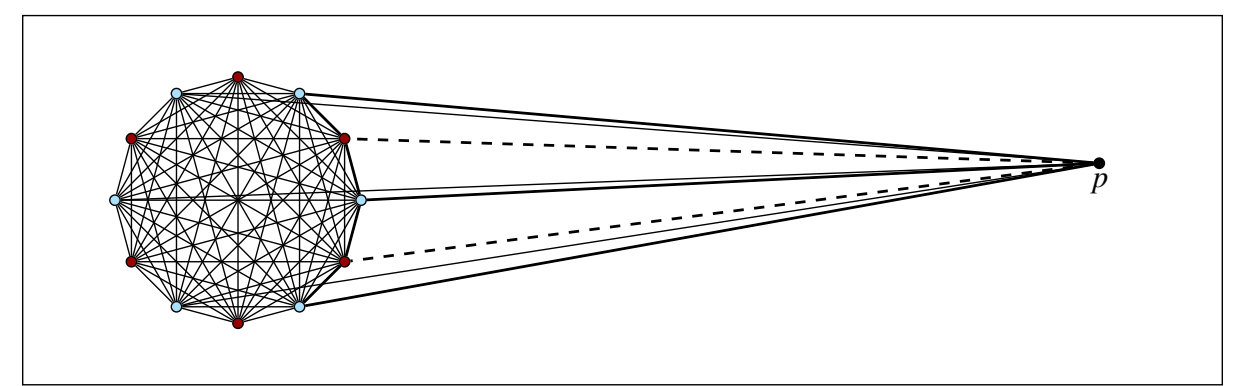

Fig. 1.2. $V\left(G_{2}\right)$ is the union of a clique $A$ of 92379 vertices, and an independent set $I$ of $\left(\begin{array}{c}92379 \\ 6\end{array}\right)$ vertices of degree 6 with distinct neighborhoods. Out of every 92379 points in general position, at least 12 are in convex position. For some drawing of $G_{2}$, we show the drawing induced on such 12 vertices comprising $A^{\prime}$ and a vertex $p \in I$ with edges to 6 vertices in $A^{\prime}$ that alternate around $\operatorname{conv}\left(A^{\prime}\right)$. In every drawing of $G_{2}$, every such choice of $A^{\prime}$ and $p$ implies the presence of at least two interior-disjoint solid quadrilaterals with non-edges inside each.

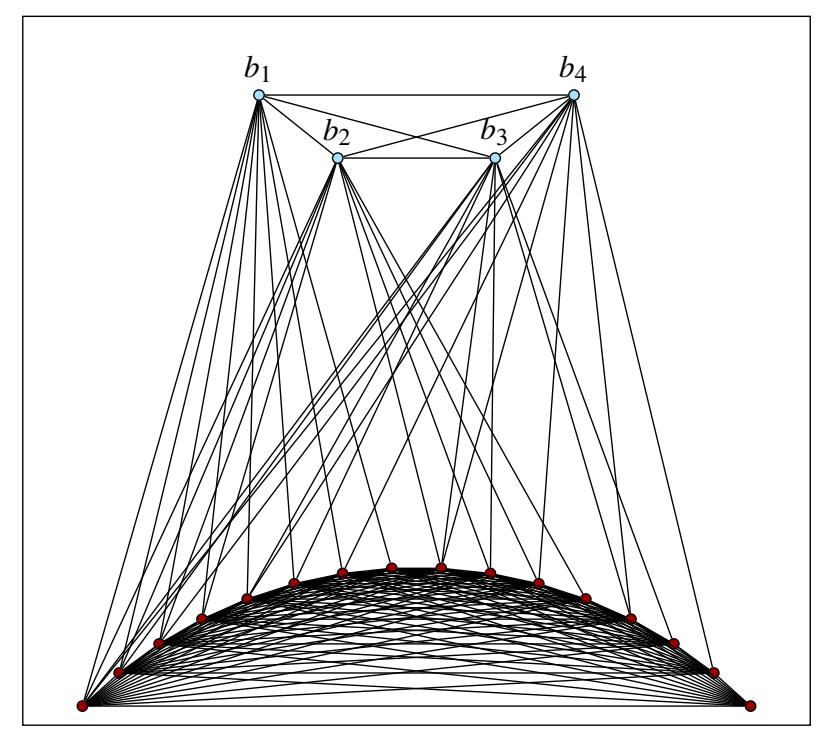

Fig. 1.3. A drawing of $G_{3}$.

Alpert et al. applied the Erdős-Szekeres convex $n$-gon theorem [6] to generalize their construction of $G_{2}$ to produce a sequence of graphs with arbitrarily large obstacle numbers. The aim of this note is to demonstrate that the existence of such graphs a simple consequence of the fact that no graph of obstacle number one contains a subgraph isomorphic to $G_{1}, G_{2}$, or $G_{3}$. In Section 2, we will show that this set of forbidden graphs allows us to utilize some extremal graph theoretic tools developed by Erdős, Kleitman, Rothschild, Frankl, Rödl, Prömel, Steger, Bollobás, Thomason, and others. They yield that the number of graphs with $n$ vertices and bounded obstacle

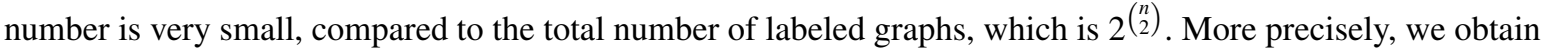


Theorem 1.2. For any fixed positive integer $h$, the number of graphs on $n$ (labeled) vertices with obstacle number at most $h$ is at most $2^{o\left(n^{2}\right)}$.

One of the unsolved questions left open in [1] was whether there exist bipartite graphs with arbitrarily large obstacle number. Since total number of labeled bipartite graphs with $n$ vertices is at least $2^{n^{2} / 4}$, the last theorem immediately implies that the answer to the above question is in the affirmative.

Theorem 1.3. For any positive integer $h$, there exists a bipartite graph with obstacle number larger than $h$.

Given any placement (embedding) of the vertices of $G$ in general position in the plane, a drawing of $G$ consists of the image of the embedding and the set of open segments connecting all pairs of points that correspond to the edges of $G$. If there is no danger of confusion, we make no notational difference between the vertices of $G$ and the corresponding points, and between the pairs $u v$ and the corresponding open segments. The complement of the set of all points that correspond to a vertex or belong to at least one edge of $G$ falls into connected components. These components are called the faces of the drawing. Notice that if $G$ has an obstacle representation with a particular placement of its vertex set, then

(1) each obstacle must lie entirely in one face of the drawing, and

(2) each non-edge of $G$ must be blocked by at least one of the obstacles.

Therefore, the problem of finding the minimum number of obstacles required for a given drawing can be reformulated as a transversal question: What is the smallest number of faces that altogether block all non-edges?

\section{Hereditary properties-The proof of Theorem 1.2}

In 1985, Erdôs, Kleitman, and Rothschild [5] proved that, as $n$ tends to infinity, the number of all $K_{\ell}$-free graphs on $n$ vertices is asymptotically equal to the number of $(\ell-1)$-partite graphs with $n$ vertices with as equal vertex classes as possible. This result was soon generalized to graphs that do not contain some fixed (not necessarily induced) subgraph $H$ [4]. Analogous questions based on the induced subgraph relation were investigated in [12], [14], and [13].

Let $\mathscr{P}$ be a graph property satisfied by infinitely many graphs. In notation, we do not distinguish between $\mathscr{P}$ and the set of all graphs that satisfy this property. The set of all graphs on $n$ labeled vertices that satisfy $\mathscr{P}$ is denoted by $\mathscr{P}^{n}$. The property $\mathscr{P}$ is called hereditary if $G \in \mathscr{P}$ implies that $G^{\prime} \in \mathscr{P}$ for every induced subgraph $G^{\prime}$ of $G$. Conversely, if $H \notin \mathscr{P}$, then $H$ is not an induced subgraph of any graph in $\mathscr{P}$. Therefore, a hereditary graph property can be characterized by its set of 'forbidden' induced subgraphs. In order to formulate an Erdős-Kleitman-Rothschild type theorem valid for any hereditary graph property, we need some definitions and notations.

A graph is $(r, s)$-colorable if its vertex set can be partitioned into $r$ blocks, out of which $s$ are cliques and every remaining block is an independent set. Let $\mathscr{C}(r, s)$ denote the set of all $(r, s)$-colorable graphs. A graph property which holds for all graphs is called trivial. Given any nontrivial hereditary graph property $\mathscr{P}$, define its coloring number as

$$
r(\mathscr{P})=\max \{r \mid \exists s: \mathscr{C}(r, s) \subseteq \mathscr{P}\} .
$$

The parameter $r(\mathscr{P})$ exists and it is at least one. Indeed, it follows from Ramsey's Theorem that $\mathscr{P}$ cannot exclude both a complete graph and an empty graph. In other words, it must be the case that $\mathscr{C}(1,0) \subseteq \mathscr{P}$ or $\mathscr{C}(1,1) \subseteq \mathscr{P}$, hence $r(\mathscr{P}) \geq 1$. Since $r(\mathscr{P})$ is strictly less than the number of vertices of any graph that does not belong to it, it is also bounded from above.

Theorem 2.1. (Bollobás, Thomason [3]) For any nontrivial hereditary graph property $\mathscr{P}$, the number of (labeled) graphs on $n$ vertices with property $\mathscr{P}$ is

$$
\left|\mathscr{P}^{n}\right|=2^{\left(1-\frac{1}{r(\mathscr{P})}+o(1)\right)\left(\begin{array}{l}
n \\
2
\end{array}\right)} .
$$

Here, it does not matter whether we count labeled or unlabeled graphs, because the corresponding quantities differ only by a factor of at most $n !=2^{O(n \log n)}$. If for some value $r$ there is no $s$ such that $\mathscr{C}(r, s) \subseteq \mathscr{P}$, then for every $r^{\prime}>r$ there is no $s$ for which $\mathscr{C}\left(r^{\prime}, s\right) \subseteq \mathscr{P}$. If we can find (2,0)-colorable, (2,1)-colorable, and (2,2)-colorable graphs, none of which has property $\mathscr{P}$, then, by the preceding observations, $r(\mathscr{P})=1$. Thus, by Theorem 2.1 , we can conclude that the number of graphs on $n$ vertices with property $\mathscr{P}$ is $2^{o\left(n^{2}\right)}$. 
The familiar term for a $(2,0)$-colorable graph is bipartite. A $(2,1)$-colorable graph consists of a clique and an independent set, possibly with edges running between them; such a graph is often called a split graph [7], [15]. A $(2,2)$-colorable graph consists of two cliques, possibly with edges running between them-its complement is bipartite.

Apply Theorem 2.1 to the hereditary property that a graph admits a 1-obstacle representation. The graphs $G_{1}$, $G_{2}$, and $G_{3}$ introduced in Section 1 are $(2,0)$-, $(2,1)$ - and $(2,2)$-colorable. Thus, in view of the fact that, according to Alpert et al. and Lemma 1.1, none of them admits a 1-obstacle representation, we can conclude that the number of all graphs on $n$ (labeled) vertices with obstacle number at most 1 is $2^{o\left(n^{2}\right)}$. In other words, Theorem 1.2 holds for $h=1$.

Denote the set of the first $n$ positive integers by $[n]$. Given $h>1$, consider a graph $G$ on the vertex set $[n]$ with obstacle number at most $h$, and fix an obstacle representation $R$ for it with $h$ obstacles $O_{1}, O_{2}, \ldots, O_{h}$. As usual, we do not distinguish between $V(G)$ and the point set corresponding to it in $R$. For each $i \in[h]$, let $G_{i}$ be the graph on $V(G)$ induced by the single obstacle $O_{i}$. It is easy to see that $G$ is a subgraph of $G_{i}$, since $O_{i}$ by itself blocks no more visibilities among $V(G)$ than do all $h$ obstacles combined. In other words, $E(G) \subseteq \cap_{i \in[h]} E\left(G_{i}\right)$. In fact, we have that $E(G)=\cap_{i \in[h]} E\left(G_{i}\right)$, since for every edge $u v \in E(G)$, the segment $u v$ avoids all obstacles specified in $R$. Let us denote by $\mathscr{G}_{h}^{n}$ the set of labeled graphs on $[n]$ with obstacle numbers at most $h$. Since every $G \in \mathscr{G}_{h}^{n}$ is uniquely determined by the above graphs $G_{1}, G_{2}, \ldots, G_{h} \in \mathscr{G}_{1}^{n}$, we have $\left|\mathscr{G}_{h}^{n}\right| \leq\left|\mathscr{G}_{1}^{n}\right|^{h}$. Using the fact that $\left|\mathscr{G}_{1}^{n}\right|=2^{o\left(n^{2}\right)}$, we can conclude that $\left|\mathscr{G}_{h}^{n}\right|=2^{o\left(n^{2}\right)}$ for any fixed $h$.

This completes the proof of Theorem 1.2.

\section{Proof of Lemma 1.1}

Let the graph $G_{3}$ consist of a set of four blue vertices $B=\left\{b_{i} \mid i \in[4]\right\}$ that induce a complete graph and a set of sixteen red vertices $R=\left\{r_{A} \mid A \subseteq[4]\right\}$ that also induce a complete graph, with additional edges between every $b_{i}$ and every $r_{A}$ with $i \in A$. We say that a polygon is solid if all its edges are edges in $G_{3}$. For three distinct points $p$, $q$, and $r$, we denote by $\angle p q r$ the union of the rays $\overrightarrow{q p}$ and $\overrightarrow{q r}$. For a point set $P$, we denote by $\operatorname{conv}(P)$ the convex hull of $P$ (the smallest convex set containing $P$ ).

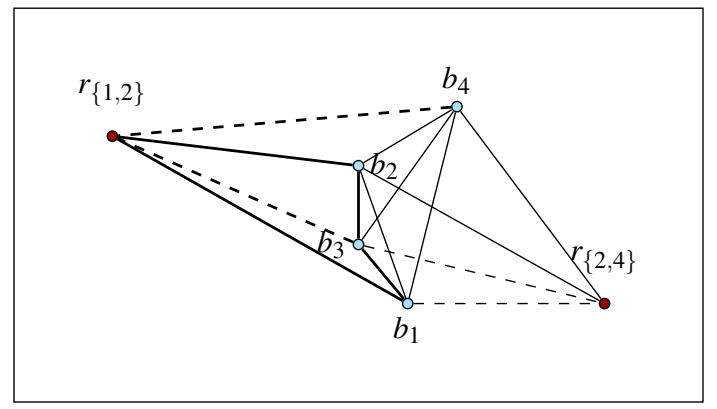

Fig. 3.1. The red vertex $r_{\{1,2\}}$ is not innocent, whereas the red vertex $r_{\{2,4\}}$ is innocent. Notice that since $r_{\{1,2\}}$ is not innocent, some solid quadrilateral (in this case $b_{1} b_{3} b_{2} r_{\{1,2\}}$ ) separates two non-edges incident on $r_{\{1,2\}}$. Therefore, distinct obstacles are required to block them.

Assume for contradiction that we are given a 1-obstacle representation of $G_{3}$. For a red vertex $r_{A}$, if there are points $p$ and $q$ such that $\angle p r_{A} q$ strictly separates $\left\{b_{i} \mid i \in A\right\}$ from the remaining blue vertices, we say that $r_{A}$ is innocent. If some red vertex $r_{A}$ is not innocent, two obstacles will be required due to the subgraph of $G_{3}$ induced on $\left\{r_{A}\right\} \cup B$, a contradiction. See Fig. 3.1.

Surely, $B$ is either in convex position or it is not. We examine the two cases separately.

Case 1: $B$ is not in convex position. Without loss of generality, $b_{4}$ is inside the triangle $\Delta b_{1} b_{2} b_{3}$. Since this is a solid triangle, the obstacle must be either inside it or outside it. We examine the two subcases separately.

Subcase 1a: The obstacle is inside $\Delta b_{1} b_{2} b_{3}$. Without loss of generality, the obstacle is inside $\Delta b_{1} b_{4} b_{3}$. This means that all non-edges must meet the interior of $\Delta b_{1} b_{4} b_{3}$. In particular, $b_{2} r_{\{1,4\}}$ and $b_{3} r_{\{1,4\}}$ must meet the interior of $\Delta b_{1} b_{4} b_{3}$. Note that every point outside an opaque convex polygon can directly see at least two vertices. 


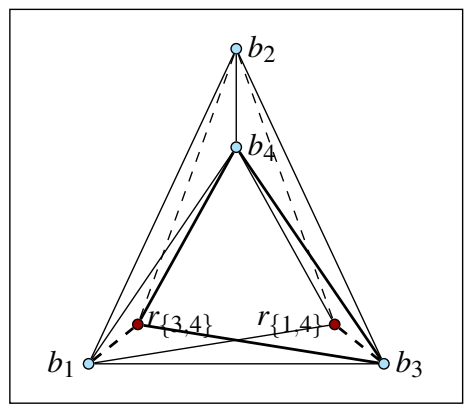

(a) Subcase 1a with $r_{\{3,4\}}$ above $b_{1} r_{\{1,4\}}$.

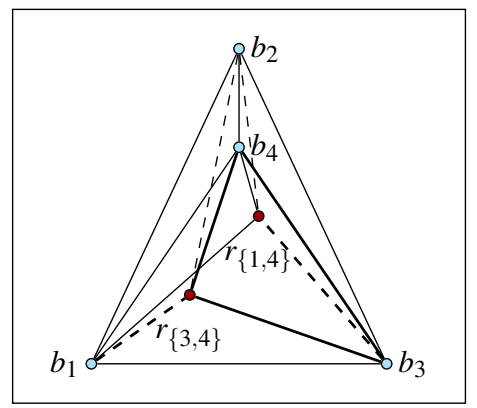

(b) Subcase 1a with $r_{\{3,4\}}$ below $b_{1} r_{\{1,4\}}$.

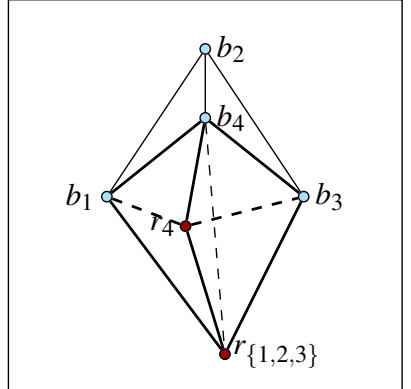

(c) Subcase $1 b$

Fig. 3.2. Case 1.

Hence, $r_{\{1,4\}}$ must be inside $\Delta b_{1} b_{4} b_{3}$, otherwise it would see at least one of $b_{2}$ or $b_{3}$ directly, i.e., the corresponding non-edge would have no intersection with the interior of $\Delta b_{1} b_{4} b_{3}$. Similarly, $r_{\{3,4\}}$ is inside $\Delta b_{1} b_{4} b_{3}$. To be innocent, $r_{\{1,4\}}$ must be in conv $\left(\angle b_{3} b_{2} b_{4}\right)$. Similarly, $r_{\{3,4\}}$ must be in conv $\left(\angle b_{4} b_{2} b_{1}\right)$. That is, the line through $b_{2}$ and $b_{4}$ separates $b_{1} r_{\{3,4\}}$ from $b_{3} r_{\{1,4\}}$.

Without loss of generality, $r_{\{1,4\}}$ is inside $\Delta b_{4} r_{\{3,4\}} b_{3}$ (otherwise, $r_{\{3,4\}}$ is inside $\Delta b_{4} r_{\{1,4\}} b_{1}$, which is symmetric). Since $b_{1} r_{\{3,4\}}$ and $b_{3} r_{\{1,4\}}$ are separated by the solid $\Delta b_{4} r_{\{3,4\}} b_{3}$, two obstacles are needed, a contradiction.

Subcase $1 b$ : The obstacle is outside of $\Delta b_{1} b_{2} b_{3}$. Hence, all non-edges must meet the outside of $\Delta b_{1} b_{2} b_{3}$. In order for $b_{4} r_{\{1,2,3\}}$ to meet the outside of $\Delta b_{1} b_{2} b_{3}, r_{\{1,2,3\}}$ must be outside of $\Delta b_{1} b_{2} b_{3}$, and without loss of generality, in conv $\left(\angle b_{1} b_{4} b_{3}\right)$.

Therefore, the obstacle is inside the convex quadrilateral $Q=b_{1} b_{4} b_{3} r_{\{1,2,3\}}$. Observe that $r_{4}$ has edges exactly to two vertices of $Q$ that comprise a diagonal of it. Since every point outside of an opaque convex polygon can directly see at least two consecutive vertices, if $r_{4}$ were outside of $Q$, then the non-edge $r_{4} b_{1}$ or the non-edge $r_{4} b_{3}$ would be outside of $Q$, requiring an obstacle outside of $Q$, a contradiction. Hence, $r_{4}$ must be inside $Q$.

Then $\angle b_{4} r_{4} r_{\{1,2,3\}}$ separates $\operatorname{conv}(Q)$ into two regions with solid boundaries that respectively contain $b_{1} r_{4}$ and $b_{3} r_{4}$. Therefore, two obstacles are needed, a contradiction.

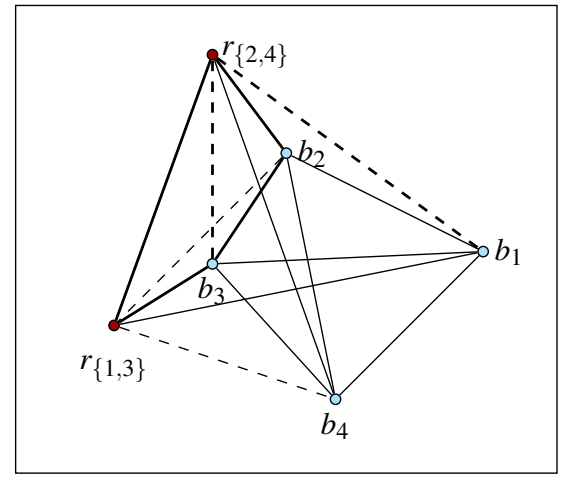

(a) Subcase $2 \mathrm{a}$

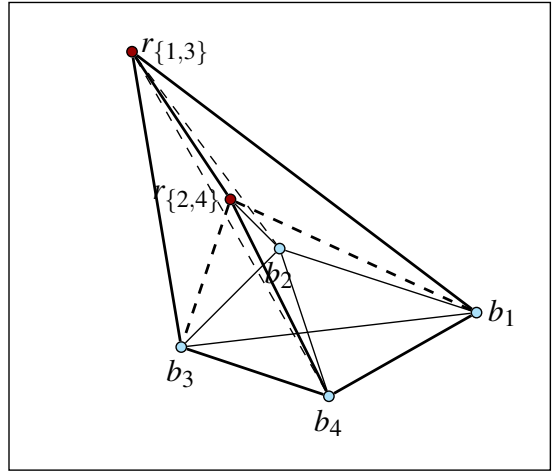

(b) Subcase $2 b$

Fig. 3.3. Case 2. The thick dashed non-edges require distinct obstacles.

Case 2: $B$ is in convex position. Without loss of generality, the bounding polygon of $B$ is $b_{1} b_{2} b_{3} b_{4}$. In order for $r_{\{1,3\}}$ and $r_{\{2,4\}}$ to be innocent,

(i) $r_{\{1,3\}}$ and $r_{\{2,4\}}$ must lie outside of $\operatorname{conv}(B)$;

(ii) for $r_{\{1,3\}}$, either $b_{1}, b_{3} \in \operatorname{conv}\left(\angle b_{2} r_{\{1,3\}} b_{4}\right)$ or $b_{2}, b_{4} \in \operatorname{conv}\left(\angle b_{1} r_{\{1,3\}} b_{3}\right)$; and

(iii) for $r_{\{2,4\}}$, either $b_{1}, b_{3} \in \operatorname{conv}\left(\angle b_{2} r_{\{2,4\}} b_{4}\right)$ or $b_{2}, b_{4} \in \operatorname{conv}\left(\angle b_{1} r_{\{2,4\}} b_{3}\right)$.

Subcase 2a: $b_{1}, b_{3} \in \operatorname{conv}\left(\angle b_{2} r_{\{1,3\}} b_{4}\right)$ and $b_{2}, b_{4} \in \operatorname{conv}\left(\angle b_{1} r_{\{2,4\}} b_{3}\right)$. Without loss of generality, the quadrilateral $b_{4} b_{1} b_{2} r_{\{1,3\}}$ is convex and has $b_{3}$ inside, and without loss of generality, the quadrilateral $b_{3} b_{4} b_{1} r_{\{2,4\}}$ is 
convex and has $b_{2}$ inside. Hence, $b_{2} b_{3} r_{\{1,3\}} r_{\{2,4\}}$ is a solid convex quadrilateral with $b_{1} r_{\{2,4\}}$ outside and $b_{3} r_{\{2,4\}}$ inside. Therefore, two obstacles are required, a contradiction.

Subcase $2 b: b_{2}, b_{4} \in \operatorname{conv}\left(\angle b_{1} r_{\{1,3\}} b_{3}\right)$ or $b_{1}, b_{3} \in \operatorname{conv}\left(\angle b_{2} r_{\{2,4\}} b_{4}\right)$. Due to symmetry, we proceed assuming the former. Without loss of generality, $Q=b_{3} b_{4} b_{1} r_{\{1,3\}}$ is a convex quadrilateral. The obstacle is inside $Q$ due to $r_{\{1,3\}} b_{4}$. In order for $b_{1} r_{\{2,4\}}$ and $b_{3} r_{\{2,4\}}$ to be blocked, $r_{\{2,4\}}$ is inside $Q$. Hence, $\angle r_{\{1,3\}} r_{\{2,4\}} b_{4}$ partitions $\operatorname{conv}(Q)$ into two regions with solid boundaries that respectively contain $b_{1} r_{\{2,4\}}$ and $r_{\{2,4\}} b_{3}$. Therefore, two obstacles are required, a contradiction.

This completes the proof of the lemma.

\section{Concluding Remark}

It was conjectured in [1] that the 10-vertex bipartite graph $G_{1}^{\prime}$ (see Fig. 3.4) has obstacle number exactly two. We showed in [11] that both $G_{1}^{\prime}$ and the 70-vertex split graph $G_{2}^{\prime}$ (see Fig. 3.5) have obstacle number at least two.

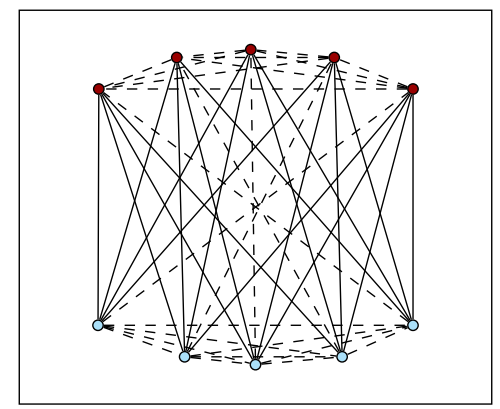

Fig. 3.4. A drawing of $G_{1}^{\prime}$ that can be completed to a 2-obstacle representation.

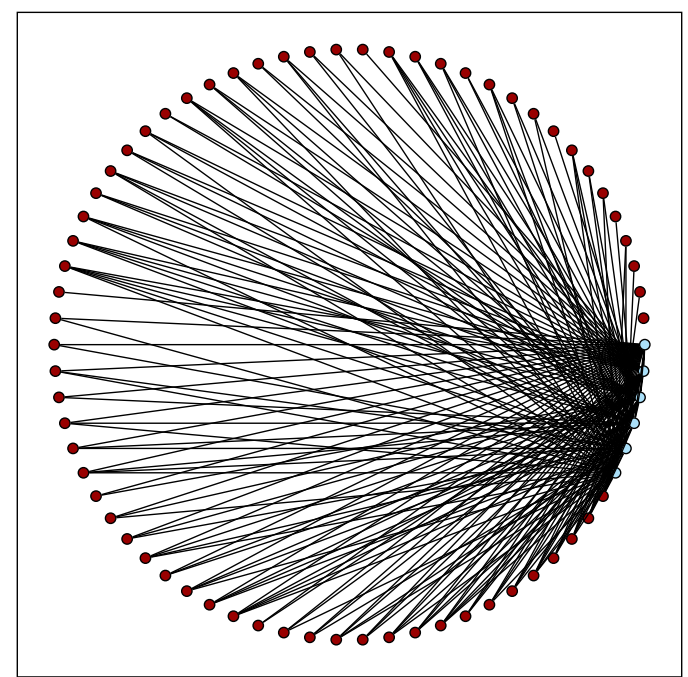

Fig. 3.5. A drawing of $G_{2}^{\prime}$, whose vertex set consists of a clique (light blue) of six vertices and an independent set (dark red) of 64 vertices with distinct neighborhoods.

Acknowledgements. We thank Filip Morić and Padmini Mukkamala for their help in finalizing this document. 


\section{References}

1. Alpert, H., Koch, C., Laison, J.: Obstacle numbers of graphs. Discrete and Computational Geometry (2009). DOI 10.1007/s00454-009-9233-8. URL http://dx.doi.org/10.1007/s00454-009-9233-8. Published at http://www.springerlink.com/content/45038g67t22463g5 (viewed on 12/26/09), 27p.

2. de Berg, M., van Kreveld, M., Overmars, M., Schwarzkopf, O.: Computational Geometry. Algorithms and Applications (2nd ed.). Springer-Verlag, Berlin (2000)

3. Bollobás, B., Thomason, A.: Hereditary and monotone properties of graphs. In: R.L. Graham, J. Nešetřil (eds.) The mathematics of Paul Erdôs vol. 2, Algorithms and Combinatorics 14, pp. 70-78. Springer, Berlin - New York (1997)

4. Erdős, P., Frankl, P., Rödl, V.: The asymptotic number of graphs not containing a fixed subgraph and a problem for hypergraphs having no exponent. Graph and Combinatorics 2, 113-121 (1986)

5. Erdős, P., Kleitman, D.J., Rothschild, B.L.: Asymptotic enumeration of $K_{n}$-free graphs. In: Colloq. int. Teorie comb., Roma, Tomo II, pp. 19-27 (1976)

6. Erdös, P., Szekeres, G.: A combinatorial problem in geometry. Compositio Math. 2, 463-470 (1935)

7. Foldes, S., Hammer, P.L.: Split graphs having Dilworth number 2. Canadian Journal of Mathematics - Journal Canadien de Mathematiques 29(3), 666-672 (1977)

8. Ghosh, S.K.: Visibility algorithms in the plane. Cambridge University Press, Cambridge (2007). DOI $10.1017 / \mathrm{CBO} 9780511543340$

9. O’Rourke, J.: Visibility. In: Handbook of discrete and computational geometry, CRC Press Ser. Discrete Math. Appl., pp. 467-479. CRC, Boca Raton, FL (1997)

10. O'Rourke, J.: Open problems in the combinatorics of visibility and illumination. In: Advances in discrete and computational geometry (South Hadley, MA, 1996), Contemp. Math., vol. 223, pp. 237-243. Amer. Math. Soc., Providence, RI (1999)

11. Pach, J., Sarioz, D.: Small $(2, s)$-colorable graphs without 1-obstacle representations (2010). URL http://arXiv.org

12. Prömel, H.J., Steger, A.: Excluding induced subgraphs: Quadrilaterals. Random Structures and Algorithms 2(1), 55-71 (1991)

13. Prömel, H.J., Steger, A.: Excluding induced subgraphs III: A general asymptotic. Random Structures and Algorithms 3(1), 19-31 (1992)

14. Prömel, H.J., Steger, A.: Excluding induced subgraphs II: extremal graphs. Discrete Applied Mathematics 44, $283-294$ (1993)

15. Tyškevič, R.I., Černjak, A.A.: Canonical decomposition of a graph determined by the degrees of its vertices. Vestsī Akad. Navuk BSSR Ser. Fìz.-Mat. Navuk 5(5), 14-26, 138 (1979)

16. Urrutia, J.: Art gallery and illumination problems. In: Handbook of computational geometry, pp. 973-1027. NorthHolland, Amsterdam (2000). DOI 10.1016/B978-044482537-7/50023-1 